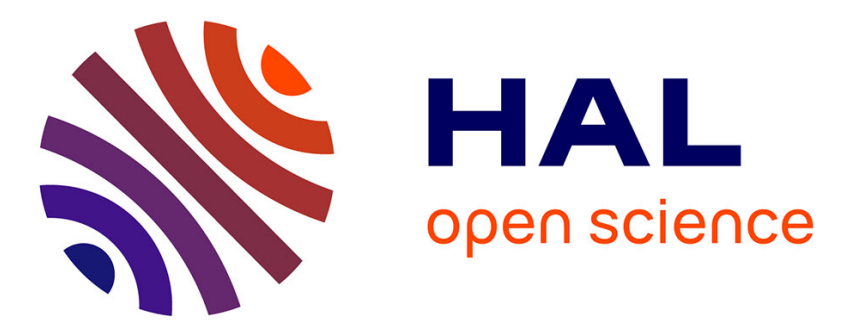

\title{
Insulin-triggered repositioning of munc18c on syntaxin- 4 in GLUT4 signalling
}

\author{
Natalie P Smithers, Conrad P Hodgkinson, Matt Cuttle, Graham J Sale
}

\section{To cite this version:}

Natalie P Smithers, Conrad P Hodgkinson, Matt Cuttle, Graham J Sale. Insulin-triggered repositioning of munc18c on syntaxin-4 in GLUT4 signalling. Biochemical Journal, 2008, 410 (2), pp.255-260. 10.1042/BJ20070802 . hal-00478829

\section{HAL Id: hal-00478829 \\ https://hal.science/hal-00478829}

Submitted on 30 Apr 2010

HAL is a multi-disciplinary open access archive for the deposit and dissemination of scientific research documents, whether they are published or not. The documents may come from teaching and research institutions in France or abroad, or from public or private research centers.
L'archive ouverte pluridisciplinaire HAL, est destinée au dépôt et à la diffusion de documents scientifiques de niveau recherche, publiés ou non, émanant des établissements d'enseignement et de recherche français ou étrangers, des laboratoires publics ou privés. 


\title{
Insulin triggered repositioning of munc18c on syntaxin-4 in GLUT4 signalling*
}

\author{
Natalie P. Smithers*, Conrad P. Hodgkinson*, Matt Cuttle, Graham J. Sale \\ School of Biological Sciences, Biomedical Sciences Building, University of \\ Southampton, Bassett Crescent East, Southampton SO16 7PX, UK
}

Short (page heading) title : Insulin regulation of munc18c syntaxin-4 interaction in vivo

Address correspondence to: Dr. Graham J Sale, School of Biological Sciences, Biomedical Sciences Building, University of Southampton, Bassett Crescent East Southampton SO16 7PX, UK. Tel: 023 8059 4307; Fax: 0238059 4459; Email:

\section{G.J.Sale@,soton.ac.uk}

* These authors contributed equally to this study. 
One of the most important action's of insulin is the stimulation of the uptake of glucose into fat and muscle cells. Crucial to this response is the translocation of the glucose transporter GLUT4 to the plasma membrane. The insulin-stimulated GLUT4 vesicle docking at the plasma membrane requires an interaction between VAMP-2 of the GLUT4 vesicle and syntaxin-4 in the plasma membrane. In the basal state, munc $18 \mathrm{c}$ is thought to preclude GLUT4 vesicle docking by inhibiting this interaction. Here, we have used fluorescence correlation spectroscopy in single living cells to show that muncl $8 \mathrm{c}$ binds to syntaxin-4 in both the basal and insulin stimulated states. We show that munc18c contains two binding sites for syntaxin-4, one of which is disrupted by insulin and a second that is activated by insulin. Insulin-triggered repositioning of munc18c on syntaxin-4 in this way in turn allows syntaxin-4 to adopt its 'open' conformation and bind VAMP-2 resulting in the docking of the GLUT4 vesicle at the cell surface. The results also demonstrate the utility of using fluorescence correlation spectroscopy in intact single living cells to elucidate cell signalling events.

Key words: Insulin, signalling, glucose uptake, GLUT4, munc18c, fluorescence correlation spectroscopy 


\section{INTRODUCTION}

The most important action of insulin is the regulation of glucose homeostasis. Activation of protein kinase $\mathrm{C}(\mathrm{PKC})^{1} \zeta$ and protein kinase $\mathrm{B}(\mathrm{PKB}$, also called $\mathrm{Akt})$ leads to the translocation of the GLUT4 glucose transporter from an intracellular vesicle to the plasma membrane. The GLUT4 vesicle contains the v-SNARE (soluble Nethylmaleimide-sensitive factor attachment protein receptor) VAMP-2 (vesicle associated membrane protein 2) and membrane binding requires interaction with the tSNARE syntaxin-4. In the basal state, the v- and t-SNARE interaction is precluded by accessory proteins such as munc18c. In un-stimulated cells the association between munc18c and syntaxin-4 is stronger than that between VAMP-2 and syntaxin-4. This blocks the GLUT4 vesicle from docking to the plasma membrane. Upon insulin stimulation the situation reverses, the association between munc18c and syntaxin-4 weakens considerably and is out-competed by the syntaxin-4 VAMP-2 interaction and thus the GLUT4 vesicle docks at the cell surface. However, the details of this mechanism, whereby insulin weakens the syntaxin-4 munc18c interaction are poorly understood [1-4].

This study utilized fluorescence correlation spectroscopy (FCS) to investigate interactions between munc18c and syntaxin-4 in intact living cells. FCS analysis indicated that munc18c did not dissociate from syntaxin-4 in response to insulin. Rather insulin triggered the repositioning of munc18c on syntaxin- 4 via an additional binding site, thus allowing VAMP-2:syntaxin-4 interaction and GLUT4 vesicle docking at the plasma membrane.

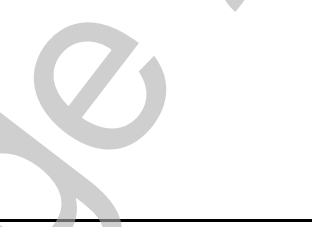

\footnotetext{
1 Abbreviations used: EYFP, enhanced yellow fluorescent protein; GLUT, glucose transporter; GST, Glutathione S Transferase; PKC, protein kinase C; SNARE, (soluble N-ethylmaleimide-sensitive factor attachment protein receptor); VAMP, vesicle associated membrane protein.
} 


\section{EXPERIMENTAL}

\section{Plasmid constructs}

The full length syntaxin-4 clone was supplied in a pCMVSport 6 vector (Invitrogen). The full length syntaxin-4 sequence was amplified by PCR using Pfu DNA Polymerase and the sequence specific forward primer 5'-ATGCGCGACAGGACCCAC-3' and reverse primer 5'-TGGTATTGGCAACCTATT-3'. The DNA product from this reaction was then used as a template for further amplification by PCR using Pfu DNA Polymerase and the forward primer (containing the EcoRI restriction site) 5'GCGGTGAATTCATGCGCGACAGGACCCAC-3' and reverse primer (containing the KpnI restriction site) 5'- TAGTGGTATTGGCAACCTACCATGGTGGCG-3'. Other constructs are described in Hodgkinson et al [5,6]. Digestion of the PCR products by EcoRI then KpnI then allowed ligation in frame with the EYFP epitope of the pEYFP-N2 vector (BD Biosciences).

\section{Transient transfection}

Cho (Cho-K1) cells were purchased from A.T.C.C. (A.T.C.C. number CCL-61; Manassas, VA, U.S.A.) and maintained at $37^{\circ} \mathrm{C}$ and $5 \% \mathrm{CO}_{2}$ on $25 \mathrm{~cm}^{2}$ tissue culture dishes in HAM-12 medium supplemented with 10\% (v/v) fetal bovine serum and 1\% (v/v) glutamine. Cho cells were transfected using Polyfect transfection reagent (Qiagen) as per the manufacturer's instructions and serum starved $24 \mathrm{~h}$ prior to FCS measurements. 3T3-L1 adipocytes were obtained and cultured as described previously [7, 8]. For electroporation, 3T3-L1 adipocytes were detached from culture dishes by incubation with $0.25 \%$ trypsin $(0.5 \mathrm{mg} / \mathrm{ml})$ and $5 \times 10^{6}$ cells mixed with $5 \mu \mathrm{g}$ of plasmid in the solution provided for the cell line Nucleofector kit V (Amaxa, Cologne, Germany). The plasmid was then introduced into the 3T3-L1 adipocytes by electroporation with the use of a Nucleofector (Amaxa) instrument according to the T-20 program. For immunoblotting, cells were extracted into $200 \mu$ lysis buffer $(62.5 \mathrm{mM}$ Tris, $\mathrm{pH} 7.4$, and $1 \%(\mathrm{w} / \mathrm{v}) \mathrm{SDS})$.

\section{FCS and confocal microscopy}

FCS and confocal microscopy were carried out with a Leica $63 \times$ water immersion 
objective and Leica SP2 confocal laser scanning microscope (Leica, Germany). Samples were excited with a $514 \mathrm{~nm} \mathrm{Ar} / \mathrm{Kr}$ laser at low-average laser power. Cells were first washed twice with $1 \mathrm{ml}$ of pre-warmed Krebs-Ringer buffer (10 mM Hepes, pH 7.4, 136 $\mathrm{mM} \mathrm{NaCl}, 4.7 \mathrm{mM} \mathrm{KCl}$ and $1.25 \mathrm{mM} \mathrm{MgSO}_{4}$ ). Cells were then incubated with $2 \mathrm{ml}$ of the same buffer. A pre-bleaching illumination period of $160 \mathrm{~s}$ was used prior to data collection. Correlation curves of $600 \mathrm{~s}$ were collected from cells and traces analysed with the Leica FCS software. Experiments were performed at room temperature to reduce the mobility of the cells. When required, cells were stimulated with $100 \mathrm{nM}$ insulin in KrebsRinger buffer for 20 minutes. Data was collected from 6 individual cells both pre and post insulin stimulation. Values are expressed as mean \pm s.e.m. The presence of the overexpressed proteins was confirmed by detection of the fluorescence by microscopy and subsequent western blotting of the cell extract (SySy, Germany; data not shown).

\section{GST pull down protocol}

BL21 cells were used to express the GST-tagged syntaxin-4 using a standard protocol supplied by Amersham Biosciences. BL21 cells over-expressing GST-tagged syntaxin-4 were lysed using Bugbuster (Novagen) and clarified extracts incubated with glutathione beads (Amersham Biosciences). After several washes with PBS, the glutathione bead syntaxin-4 complex was exposed to cell extracts prepared from Cho cells over-expressing Xpress-tagged munc $18 \mathrm{c}$ constructs. After 4 hours of constant agitation at $4^{\circ} \mathrm{C}$, complexes were washed 3 times with PBS and then resuspended in a small volume of PBS.

\section{Immunoprecipitation}

Cells were extracted into lysis buffer (50mM Tris $\mathrm{pH} 7.5,150 \mathrm{mM} \mathrm{NaCl,} \mathrm{1 \%} \mathrm{v/v} \mathrm{Sigma}$ Protease and Phosphatase Inhibitor cocktails) to give a $500 \mu \mathrm{l}$ final volume $(500 \mu \mathrm{g})$. Coimmunoprecipitation was carried out by incubating $500 \mu \mathrm{g}$ cell lysate with a monoclonal Xpress antibody ( $5 \mu 1$, Invitrogen) and protein G:protein A beads (50:50, 20 $\mu \mathrm{L}$, Sigma). After $5 \mathrm{~h}$ continuous gentle agitation at $4{ }^{\circ} \mathrm{C}$, the beads were collected by pulse spin and then washed 3 times in lysis buffer after which they were resuspended in PBS. 


\section{Immunoblotting}

Samples were resolved by SDS-PAGE [7, 8] and transferred to a nitrocellulose filter. The membranes were probed with various primary antibodies followed by incubation with horseradish peroxidase-conjugated secondary antibodies. Primary antibodies [GST (NEB), munc18c (BD Biosciences), living colours antibody (SySy, Germany), syntaxin-4 (SySy, Germany) Xpress (Invitrogen) and 80K-H (BD Biosciences)] were used according to the manufacturer's instructions. Blots were developed using the ECL $($ system according to the manufacturer's instructions (Amersham Biosciences)

\section{RESULTS}

The FCS data from this study was fitted using a 3D Gaussian triplet calculation (Leica software) and the tauD1 value calculated in $\mu$ s. TauD1 represents the average time the EYFP-tagged construct spends in the observation volume and thus can be called a diffusion coefficient. A larger diffusion coefficient corresponds to a longer time spent in the observation volume and hence relates to a slower moving molecule. Knowing the average speed of the protein of interest in vivo provides valuable insight into its behaviour.

To investigate insulin triggered interactions between munc18c, syntaxin- 4 and other proteins in vivo within intact cells one or two of the proteins, or non-binding constructs, were expressed. In all experiments cells were serum starved $24 \mathrm{~h}$ prior to data collection and were bleached for $120 \mathrm{~s}$ prior to data collection in order to provide optimal signal to noise for FCS (this typically being 10-100 fluorescent molecules in the focal volume). Levels of the constructs were determined by immunoblotting. Analysis of the immunoblots showed that expression levels were similar for all the constructs used in this study (data not shown). Cho cells and 3T3 L1 adipocytes were employed in this study. Cho cells possess GLUT4 storage vesicles which translocate to the plasma membrane in response to insulin and have been frequently used to investigate the mechanism whereby insulin stimulation promotes GLUT4 translocation to the plasma membrane. 3T3 L1 adipocytes were utilised as a truly insulin responsive cell type. The two cell types yielded similar results. 
Insulin did not affect the tauD1 value of the EYFP-tagged full length munc18c construct when expressed alone (Tables 1 and 2). When the EYFP-tagged munc18c construct was co-expressed with syntaxin-4 the diffusion co-efficient of EYFP-tagged full length munc18c was markedly increased compared to the single transfection both pre-insulin and post-stimulation Tables 1 and 2). The effects of co-expressing syntaxin-4 were highly significant with $\mathrm{p}$ values of $<0.01$ and indicate that munc $18 \mathrm{c}$ binds syntaxin4 both in the presence and absence of insulin. Example FCS traces are shown in Fig. 1.

To confirm this, we next determined the diffusion co-efficient of EYFP-tagged syntaxin-4. Insulin stimulation had no effect on the diffusion co-efficient of EYFP-tagged syntaxin-4 (Table 1). However, when full length munc18c was co-expressed, the diffusion coefficients for EYFP-tagged syntaxin-4 were again markedly increased both pre insulin and post insulin stimulation (Table 1). These increases are virtually identical to those for the EYFP-tagged full length munc18c construct in the EYFP-tagged full length munc18c and syntaxin-4 transfections. Again insulin had no effect on the diffusion coefficients.

To further define the binding of munc $18 \mathrm{c}$ to syntaxin-4, we employed a truncation construct of munc18c, namely munc18c 295, which lacks the N-terminal 295 amino acids. GST pull-down assays in our laboratory showed that this construct is unable to bind to syntaxin- 4 when expressed in the non insulin responsive Cos- 1 cell line (data not shown). This is in accordance with other published data [9] which states that the Nterminal 139 amino acids of munc18c bind to syntaxin-4. The diffusion coefficient for the EYFP-tagged munc18c 295 was not significantly affected by insulin stimulation (Tables 1 and 2). The co-expression of syntaxin-4 with EYFP-tagged munc18c 295 had no statistically significant effect on the diffusion co-efficient of the EYFP-tagged munc18c 295 construct in the basal state. In contrast, insulin stimulation caused a major increase in the diffusion co-efficient of the EYFP-tagged munc18c 295 construct (Tables 1 and 2; Fig. 1). This effect was highly significant with a $p$ value of $<0.01$ and indicated that munc18c 295 only binds syntaxin-4 in the insulin-stimulated state. These results suggest that munc18c has two binding sites for syntaxin-4. One that lies between residues 1-295, as has been shown previously, and one that lies between residues 295-592 which binds syntaxin-4 in the presence of insulin. 
To further confirm that insulin was working in the cells and to help validate the FCS approach, the interaction of munc18c with $80 \mathrm{~K}-\mathrm{H}$ was analyzed. $80 \mathrm{~K}-\mathrm{H}$ is believed to be an upstream regulator of munc $18 \mathrm{c}$ that is crucial for delivering an insulin signal to the GLUT4 translocation machinery [5, 6]. Co-expression of EYFP-tagged full length munc $18 \mathrm{c}$ with $80 \mathrm{~K}-\mathrm{H}$ resulted in insulin eliciting a major increase in the mobility of the full length EYFP-tagged munc18c of approx. 2 fold ( $\mathrm{p}<0.01$; Tables 3 and 4$)$. To test if this effect was dependent on the ability of $80 \mathrm{~K}-\mathrm{H}$ to bind munc $18 \mathrm{c}$, a truncation construct of munc18c, namely, EYFP-tagged munc18c 338 was employed. This construct is unable to bind $80 \mathrm{~K}-\mathrm{H}$ and inhibits insulin stimulated GLUT4 translocation to a greater extent than full length munc18c. As with the EYFP-tagged full length munc18c, insulin did not affect the diffusion coefficient of EYFP-tagged munc18c 338 when expressed alone. Importantly, when EYFP-tagged munc18c 338 and 80K-H were co-expressed insulin was no longer able to trigger a change in the speed of the munc $18 \mathrm{c}$ construct (Tables 3 and 4). As a control, a less truncated version of munc18c was employed, namely, EYFP-tagged munc18c 295. This construct still has the ability to bind $80 \mathrm{~K}-\mathrm{H}$ and like the full length munc18c to inhibit GLUT4 translocation when over-expressed. Insulin did not affect the mobility of the EYFP-tagged munc18c 295 construct when expressed alone. However, when EYFP-tagged munc18c 295 and $80 \mathrm{~K}-\mathrm{H}$ were co-expressed insulin stimulation caused an approx 2-fold increase in the speed of EYFP-tagged munc18c 295.

To further test the nature of the interactions between munc18c and syntaxin-4, coimmunopreciptation experiments were performed. For this Xpress-tagged munc18c 295 or full length munc18c constructs were expressed in Cho cells and the cells incubated with and without insulin. Only with the insulin-stimulated cells did syntaxin-4 coimmunopreciptate with the Xpress-tagged 295 construct whereas with both unstimulated and insulin-stimulated cells syntaxin-4 co-immunopreciptated with the Xpress-tagged full length munc18c construct (Fig. 2). As expected, 80-KH co-immunoprecipitated with munc18c 295 and syntaxin-4 from insulin-stimulated but not unstimulated cells (Fig. 3). Crucially this co-immunopreciptation data supports by a different method the FCS data.

The above results establish that full length munc18c can bind syntaxin-4 both in the presence and absence of insulin, whereas munc18c 295 can only bind syntaxin- 4 in the presence of insulin. Munc18c 295 lacks the well established binding site for syntaxin- 
4 located in the $\mathrm{N}$-terminus of the protein. The results above thus indicate that munc18c contains a second syntaxin- 4 binding site. To help define the nature of the second syntaxin binding site, further co-immunoprecipitation experiments were performed by more extensively truncating munc18c. For this various Xpress-tagged munc $18 \mathrm{c}$ truncation constructs were expressed in Cho cells and the cells incubated with insulin. Cell extracts were then incubated with GST-tagged syntaxin-4 glutathione beads. As expected the Xpress-tagged munc18c 295 construct was pulled down by the GST-tagged syntaxin-4 glutathione beads. In contrast the Xpress-tagged munc18c 308, 326 and 338 constructs were not pulled down by the GST-tagged syntaxin-4 glutathione beads (Fig 4). This indicates that key residues involved in forming the second binding site are located between amino acids 295 and 308 of munc18c.

\section{DISCUSSION}

Insulin regulates glucose homeostasis predominantly by causing a rapid increase in glucose uptake in muscle and adipose cells via the translocation of the GLUT4 vesicle to the cell surface. GLUT4 vesicle trafficking is similar to that seen in synaptic vesicles. The GLUT4 vesicle contains the V-SNARE VAMP-2 which binds to its cognate tSNARE receptor syntaxin-4 in the plasma membrane [10-12]. Insulin regulates the VAMP-2:syntaxin-4 binding by virtue of accessory proteins, the most important of which is munc $18 \mathrm{c}$.

There have however been few studies conducted to elucidate the exact role of munc18c in GLUT4 vesicle exocytosis. Munc18c has been shown to inhibit GLUT4 vesicle exocytosis and yet to be also essential for this process. In vitro assays have shown that in the basal state munc18c binds to syntaxin-4 with higher affinity than VAMP-2. This prevents VAMP-2 from binding to syntaxin-4 and hence inhibits GLUT4 vesicle docking at the plasma membrane $[11,13,14]$. These same in vitro assays also showed that when insulin stimulation decreases the binding strength of munc18c towards syntaxin-4, the VAMP-2 interaction with syntaxin-4 becomes relatively stronger. This allows the GLUT4 vesicle to bind to the plasma membrane. Due to the in-vitro nature of these assays the mechanistic detail in vivo remained un-categorized. Several scenarios would fit this data in vivo. Either munc18c would dissociate from syntaxin-4 in response 
to insulin or weaker binding association would not cause munc18c dissociation but rather allow syntaxin-4 to undergo a conformational change allowing syntaxin-4 to bind VAMP-2.

In our study, FCS has been utilized to investigate munc18c biology in intact living cells. Co-expression of EYFP-tagged full length munc18c with syntaxin-4 resulted in a significant increase in the diffusion coefficient of the EYFP-tagged full length munc18c in the absence of insulin stimulation. This slow down in the mobility of munc18c is expected as munc18c is known to bind syntaxin-4. The insulin-independent binding between munc18c and syntaxin-4 was confirmed using EYFP-tagged syntaxin-4 in the presence and absence of co-expression of munc18c. If insulin caused munc18c to physically dissociate from syntaxin-4, then insulin stimulation would be expected to increase the mobility of the EYFP-tagged full length munc18c. However, the diffusion co-efficient of EYFP-tagged munc18c did not change in response to insulin. The same result was seen when the diffusion co-efficient of EYFP-tagged syntaxin-4 was measured in the presence of munc18c. This in vivo data clearly shows that munc18c is not released from syntaxin- 4 in response to insulin. Munc18c belongs to the $\mathrm{nSec} 1$ family of proteins. Yeast Sec1 proteins have been the most heavily studied. Several mechanisms appear to be used for interaction between Sec1 proteins and syntaxins. Sec1 only binds to the yeast syntaxin Sso 1p when the complete SNARE complex is formed [15]. The structure of the neuronal SM protein, $\mathrm{nSec} 1$, bound to the closed conformation of Syntaxin 1a has been interpreted as an intermediate required to convert syntaxin to the "open" conformation, which then assembles with the other SNAREs to form the fusogenic SNARE complex [16]. An indirect syntaxin-binding mode describes the interaction of the yeast vacuolar $\mathrm{SM}$ protein, Vps33p, which is part of a large protein complex that binds to the cognate syntaxin, Vam3p [17]. Indeed null mutations in the genes for the SM proteins cause dramatic reductions in vesicle exocytosis, suggesting that these proteins are essential for normal SNARE function [18]. The munc18c-Syntaxin-4 complex has been shown to be specifically required for insulin-stimulated GLUT4 vesicle fusion and munc18c has been described as a positive fusogenic protein [19]. Our FCS data agrees with these findings. If munc18c is not actually released by syntaxin-4 some change on munc18c must allow syntaxin-4 to bind VAMP-2. 
To test if munc18c has a binding site for syntaxin-4 distinct from the well known site in the N-terminal portion of the protein, the EYFP-tagged munc18c 295 construct which lacks the site was employed. The mobility of the EYFP-tagged munc18c 295 construct was similar in the presence and absence of co-expressed syntaxin- 4 in the absence of insulin. This indicates that, unlike full length munc18c, the munc $18 \mathrm{c} 295$ construct does not associate with syntaxin-4 in the basal state and that the $\mathrm{N}$-terminal region is responsible for binding syntaxin-4 in the basal state. Upon insulin stimulation of cells co-expressing the EYFP-tagged munc18c 295 construct and syntaxin-4 there was a marked increase in the diffusion coefficient of the EYFP-tagged munc18c 295 construct. This indicates that the munc18c 295 construct was able to associate with syntaxin- 4 in the insulin stimulated state and that munc $18 \mathrm{c}$ contains a second binding site for syntaxin- 4 that is outside the $\mathrm{N}$-terminal region. Thus insulin may trigger a conformational change in munc $18 \mathrm{c}$ that results in it repositioning on syntaxin-4 and in turn allowing syntaxin-4 to adopt its 'open' conformation and bind VAMP-2. Very recent data from D'AndreaMerrins et al [21] supports our view that munc18c has more than one binding site on syntaxin-4.

To analyze how the insulin signal may be delivered to munc18c to induce such a conformational change experiments were conducted with $80 \mathrm{~K}-\mathrm{H}$. Recent evidence suggests that $80 \mathrm{~K}-\mathrm{H}$ links insulin signalling to the machinery controlling GLUT4 exocytosis through an insulin triggered association with munc18c [5, 6]. For this, EYFPtagged full length munc $18 \mathrm{c}$ was expressed in the presence and absence of $80 \mathrm{~K}-\mathrm{H}$. While insulin did not affect the mobility of EYFP-tagged full length munc18c when expressed alone, in the presence of $80 \mathrm{~K}-\mathrm{H}$ insulin induced a large increase in the mobility of the EYFP-tagged full length munc18c. Use of various constructs of munc18c showed that this increase in speed of munc18c was critically dependent on the presence of an intact $80 \mathrm{~K}-\mathrm{H}$ binding site on munc18c. These results indicate that $80 \mathrm{~K}-\mathrm{H}$ interacts with munc $18 \mathrm{c}$ in intact living cells. How then does insulin trigger $80 \mathrm{~K}-\mathrm{H}$ to increase the speed of munc18c if, as shown above, munc18c remains associated with syntaxin-4? One way that the mobility of munc18c can increase is if there is a decrease in the tethering of the syntaxin-4 complex with other known interactors such as synip or tomosyn or other scaffolding proteins. In this model insulin would break or reduce these interactions. This 
model is shown in Fig. 5. However, we did not observe an increase in mobility of syntaxin-4 when cells co-expressing munc18c and syntaxin-4 were stimulated with insulin. This is likely because the availability of the other interactors was limiting and that most of the overexpressed syntaxin- 4 was not tethered in the first place. Tyrosine phosphorylation of munc18c has been shown to promote VAMP-2 binding to syntaxin-4 and this provides a further mechanism of delivering an insulin signal to munc18c [20].

The above conclusions, based on differences in the diffusion coefficients between full length munc18c and munc18c 295 (Tables 1 and 2), suggest that in unstimulated cells the latter does not interact with syntaxin-4, whereas association is enhanced with syntaxin-4 in insulin-stimulated cells. This is a critical result that raises the concept that reorganising the molecular interaction between munc18c and syntaxin-4 serves as a gating mechanism that permits interaction between SNARE proteins. To underpin this finding it was important to substantiate these interactions by another approach. For this Xpress-tagged munc18c 295 or full length munc18c constructs were expressed in Cho cells. If the proposition holds then only in insulin-stimulated cells should syntaxin-4 coimmunoprecipitate with munc18c 295. Western blots in Fig. 2 show that this was indeed the case. Additionally, if the proposition is correct full length munc18c should not undergo any change in association with syntaxin-4 upon insulin treatment. Again this was found to be the case (Fig. 2). These results corroborate the repositioning hypothesis.

In conclusion, we have identified that insulin does not trigger the release of munc18c from syntaxin-4 in intact single living cells. Rather munc18c appears to contain two binding sites for syntaxin-4, one of which is disrupted by insulin and the other exposed by insulin. Thus insulin may elicit a repositioning of munc18c on syntaxin- 4 which in turn allows syntaxin-4 to adopt its 'open' conformation and bind VAMP-2.

\section{REFERENCES}

1. Ishiki, M., and Klip, A. (2005) Minireview: recent developments in the regulation of glucose transporter-4 traffic: new signals, locations, and partners. Endocrinology 146, 5071-5078

2. Kanzaki, M., Mora, S., Hwang, J.B., Saltiel, A.R., and Pessin, J.E. (2004) 
Atypical protein kinase $\mathrm{C}$ (PKCzeta/lambda) is a convergent downstream target of the insulin-stimulated phosphatidylinositol 3-kinase and TC10 signaling pathways. J. Cell. Biol. 164, 279-290

3. Patel, N., Huang, C., and Klip, A. (2006) Cellular location of insulin-triggered signals and implications for glucose uptake. Pflugers Arch. 451, 499-510

4. Watson, R.T. and Pessin, J.E. (2001) Subcellular compartmentalization and trafficking of the insulin-responsive glucose transporter, GLUT4. Experimental Cell Research 271, 75-83

5. Hodgkinson, C.P., Mander, A., and Sale, G.J. (2005) Identification of 80K-H as a protein involved in GLUT4 vesicle trafficking. Biochem. J. 388, 785-793

6. Hodgkinson, C.P., Mander, A., and Sale, G.J. (2005) Protein kinase-zeta interacts with munc18c: role in GLUT4 trafficking. Diabetologia 48, 1627-1636

7. Sale, E.M., Atkinson, P.G., and Sale, G.J. (1995) Requirement of MAP kinase for differentiation of fibroblasts to adipocytes, for insulin activation of p90 S6 kinase and for insulin or serum stimulation of DNA synthesis. EMBO J 14, 674-684

8. Arnott, C.H., Sale, E.M., Miller, J., and Sale, G.J. (1999) Use of an antisense strategy to dissect the signalling role of protein-tyrosine phosphatase alpha. J Biol Chem 274, 26105-26112

9. Grusovin, J., Stoichevska, V., Gough, K.H., Nunan, K., Ward, C.W., and Macaulay, S.L. (2000) Definition of a minimal munc18c domain that interacts with syntaxin 4. Biochem. J. 350, 741-746

10. Kawanishi, M., Tamori, Y., Okazawa, H., Araki, S., Shinoda, H., and Kasuga, M. (2000) Role of SNAP23 in insulin-induced translocation of GLUT4 in 3T3-L1 adipocytes. Mediation of complex formation between syntaxin4 and VAMP2. J Biol Chem. 275, 8240-8247

11. Kanda, H., Tamori, Y., Shinoda, H., Yoshikawa, M., Sakaue, M., Udagawa, J., Otani, H., Tashiro, F., Miyazaki, J., and Kasuga, M. (2005) Adipocytes from Munc18c-null mice show increased sensitivity to insulin-stimulated GLUT4 externalization. J Clin Invest. 115, 291-301

12. Chang L, Chiang S.H. and Saltiel, A.R. (2004) Insulin signaling and the regulation of glucose transport. Mol Med. 10, 65-71. 
13. Thurmond, D.C., Ceresa, B.P., Okada, S., Elmendorf, J.S., Coker, K., and Pessin, J.E. (1998) Regulation of insulin-stimulated GLUT4 translocation by Munc18c in 3T3L1 adipocytes. J Biol Chem. 273, 33876-33883

14. Tamori, Y., Kawanishi, M., Niki, T., Shinoda, H., Araki, S., Okazawa, H., and Kasuga, M. (1998) Inhibition of insulin-induced GLUT4 translocation by Munc18c through interaction with syntaxin4 in 3T3-L1 adipocytes. J Biol. Chem. 273, 19740-19746

15. Togneri, J., Cheng, Y.S., Munson, M., Hughson, F.M., and Carr, C.M. (2006) Specific SNARE complex binding mode of the Sec1/Munc-18 protein, Sec1p. Proc Natl Acad Sci U S A. 103, 17730-17735

16. Misura, K.M., Scheller, R.H., and Weis, W.I. (2000) Three-dimensional structure of the neuronal-Sec1-syntaxin 1a complex. Nature. 404, 355-362

17. Seals, D.F., Eitzen, G., Margolis, N., Wickner, W.T., and Price A. (2000) A $\mathrm{Ypt} / \mathrm{Rab}$ effector complex containing the Sec1 homolog Vps33p is required for homotypic vacuole fusion. Proc Nat1 Acad Sci U S A. 97, 9402-9407.

18. Hosono, R., Hekimi, S., Kamiya, Y., Sassa, T., Murakami, S., Nishiwaki, K., Miwa, J., Taketo, A., and Kodaira, K.I. (1992) The unc-18 gene encodes a novel protein affecting the kinetics of acetylcholine metabolism in the nematode Caenorhabditis elegans. J Neurochem. 58, 1517-1525

19. Thurmond, D.C. and Pessin, J.E. (2000) Discrimination of GLUT4 vesicle trafficking from fusion using a temperature-sensitive Munc18c mutant. EMBO J. 19, 3565-3575

20. Oh, E., and Thurmond, D.C. (2006) The stimulus-induced tyrosine phosphorylation of Munc18c facilitates vesicle exocytosis. J Biol Chem. 281, $17624-17634$

21. D'Andrea-Merrins, M., Chang, L., Lam, A.D., Ernst, S.A. and Stuenkel, E.L. (2007) Munc18c Interaction with Syntaxin 4 Monomers and SNARE Complex Intermediates in GLUT4 Vesicle Trafficking. J. Biol. Chem. 282, 16553-16566

This work was funded by grants from the BBSRC and Diabetes UK 


\section{FIGURE LEGENDS}

Figure 1 Example FCS traces showing that syntaxin-4 reduces the diffusion coefficient of munc18c 295, but not full length munc18c, in an insulin-dependent manner

Syntaxin-4 was expressed with the EYFP-tagged full length munc18c construct (A and B) or the EYFP-tagged munc18c 295 construct (C and D) which lacks the well characterized syntaxin- 4 binding site. Cells were serum starved for $24 \mathrm{~h}$, stimulated with (B and D) or without (A and C) $10^{-7} \mathrm{M}$ insulin for $20 \mathrm{~min}$ and FCS data collected. Note the shift to the right of the curve in panel $\mathrm{D}$ compared with that in panel $\mathrm{C}$ demonstrating an insulin-stimulated increase in the diffusion coefficient of the EYFP-tagged munc18c 295 construct; this corresponds to a reduction in mobility of the EYFP-tagged munc18c 295 construct. When full length munc18c was employed, insulin-stimulation did not shift the curve. The original FCS traces are shown.

Figure 2 Syntaxin-4 interacts with munc18c 295 in an insulin-dependant manner and with full length munc18c in an insulin-independent manner

Cho cells, over-expressing Xpress-tagged full length munc18c (A) or Xpress-tagged munc18c 295 (B), were serum-starved for $24 \mathrm{~h}$ and then incubated for $20 \mathrm{~min}$ in the presence or absence of $100 \mathrm{nM}$ insulin. Xpress-tagged munc18c proteins were immunoprecipitated from cell lysates $(0.5 \mathrm{mg}$ of protein) and the immunoprecipitates were washed extensively. Proteins in the immunocomplex were immuno-blotted with an anti-syntaxin-4 antibody (upper panels, lanes 3 and 4) or anti-munc18c antibody as a control (lower panels, lanes 3 and 4). Control immunoprecipitations containing an equal amount of cell lysate and Protein $\mathrm{A} / \mathrm{G}$ beads but omitting the antibody were also performed (lanes 1 and 2). The results are representative of two independent experiments.

Figure 3 Immunopreciptates of munc18c 295 from insulin-stimulated but not unstimulated cells contain $80 \mathrm{~K}-\mathrm{H}$

Cho cells, over-expressing Xpress-tagged munc18c 295, were serum-starved for $24 \mathrm{~h}$ and then incubated for $20 \mathrm{~min}$ in the presence or absence of $100 \mathrm{nM}$ insulin. Xpress-tagged munc18c 295 was immunoprecipitated from cell lysates $(0.5 \mathrm{mg}$ of protein) and the 
immunoprecipitates were washed extensively. Proteins in the immunocomplex were immuno-blotted with either antibodies against syntaxin-4 (A), 80K-H (B) or munc18c (C) as a control (lanes 3 and 4). Control immunoprecipitations containing an equal amount of cell lysate and Protein A/G beads but omitting the antibody were also performed (lanes 1 and 2). The results are representative of two independent experiments.

Figure 4 Munc18c 295, but munc18c 308, 326 and 338 interact with syntaxin-4 Cho cells, over-expressing the Xpress-tagged munc18c constructs, were serum-starved for $24 \mathrm{~h}$ and then incubated for $20 \mathrm{~min}$ in the presence of $100 \mathrm{nM}$ insulin. GST-tagged syntaxin-4 was produced in bacteria, and after purification, coupled to Glutathione beads. The GST-tagged syntaxin-4 glutathione beads were exposed to the Cho cell extracts ( 0.5 mg of protein) containing the Xpress-tagged munc18c constructs. After incubation, the complexes were washed extensively. Complexes were probed with an anti-Xpress

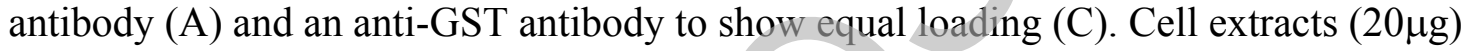
were probed with an anti-Xpress antibody to confirm expression of the constructs (B).

\section{Figure 5 Hypothetical model showing how insulin triggers the repositioning of munc18c on syntaxin-4}

Upstream signaling molecules including $\mathrm{PKC} \zeta$ and $80 \mathrm{~K}-\mathrm{H}$ relay the insulin signal to munc18c. Activated munc 18c then repositions itself on syntaxin-4 thereby triggering syntaxin-4 to adopt the "open" formation enabling it to bind VAMP-2 of the GLUT4 vesicle. Breakage of interactions between syntaxin- 4 and other known interactors such as synip, tomosyn, SNAP-23 or other scaffolders may account for the observed increase in munc $18 \mathrm{c}$ mobility. 
Table 1 Action of insulin on the diffusion coefficients of munc18c and syntaxin-4 in cho cells

The indicated EYFP-tagged constructs were expressed in the presence or absence of the indicated interactor. Cells were serum starved for $24 \mathrm{~h}$, stimulated with or without $10^{-7} \mathrm{M}$ insulin for $20 \mathrm{~min}$, FCS data collected and diffusion coefficients measured ( $\mu \mathrm{s})$. Results are mean \pm s.e.m. for 3 separate experiments each using 6 separate cells. * $p<0.01$ versus absence of the interactor. MFL, munc18c full length; M295, munc18c 295.

\begin{tabular}{llll}
\hline EYFP & Interactor & No insulin & Insulin \\
\hline MFL & & $526 \pm 41$ & $592 \pm 78$ \\
MFL & Syntaxin-4 & $795 \pm 69^{*}$ & $804 \pm 61^{*}$ \\
M295 & & $576 \pm 64$ & $489 \pm 36$ \\
M295 & Syntaxin-4 & $619 \pm 34$ & $821 \pm 32^{*}$ \\
Syntaxin-4 & & $459 \pm 54$ & $491 \pm 71$ \\
Syntaxin-4 & MFL & $720 \pm 59 *$ & $789 \pm 88^{*}$ \\
\hline
\end{tabular}

Table 2 Action of insulin on the diffusion coefficients of munc18c and syntaxin-4 in 3T3-L1 adipocytes

The indicated EYFP-tagged constructs were expressed in the presence or absence of the indicated interactor. Cells were serum starved for $24 \mathrm{~h}$, stimulated with or without $10^{-7} \mathrm{M}$ insulin for $20 \mathrm{~min}$, FCS data collected and diffusion coefficients measured $(\mu \mathrm{s})$. Results are the mean \pm s.e.m for 3 separate experiments each using 6 separate cells. ${ }^{*} \mathrm{p}<0.01$ versus absence of the interactor. MFL, munc18c full length; M295, munc18c 295.

\begin{tabular}{llll}
\hline EYFP & Interactor & No Insulin & Insulin \\
\hline MFL & & $480 \pm 25$ & $507 \pm 17$ \\
MFL & Syntaxin-4 & $776 \pm 71^{*}$ & $772 \pm 27^{*}$ \\
M295 & & $501 \pm 69$ & $561 \pm 58$ \\
M295 & Syntaxin-4 & $493 \pm 62$ & $748 \pm 57^{*}$ \\
\hline
\end{tabular}


Table 3 Insulin increases the mobility of munc18c in an $80 \mathrm{~K}-\mathrm{H}$ dependant manner in cho cells

The indicated EYFP-tagged munc18c constructs were expressed in the presence or absence of $80 \mathrm{~K}-\mathrm{H}$. Cells were serum starved for $24 \mathrm{~h}$ and stimulated with or without $10^{-7}$ M insulin for $20 \mathrm{~min}$. FCS data was collected, diffusion coefficients measured and the effect of stimulation by insulin on the mobility (speed) of the EYFP construct determined. Each value is the ratio of the diffusion coefficients (absence of insulin / presence of insulin). Results are mean \pm s.e.m. for 3 separate experiments each using 6 separate cells. ${ }^{*} \mathrm{p}<0.01$ versus no $80 \mathrm{~K}-\mathrm{H}$. MFL, munc18c full length, M295, munc18c 295; M338, munc18c 338.

\begin{tabular}{lcc}
\hline EYFP & No $80 \mathrm{~K}-\mathrm{H}$ & $80 \mathrm{~K}-\mathrm{H}$ \\
\hline & \multicolumn{1}{c}{ Effect of insulin on mobility } \\
MFL & $0.83 \pm 0.07$ & $1.75 \pm 0.18^{*}$ \\
M295 & $1.1 \pm 0.01$ & $1.96 \pm 0.27^{*}$ \\
M338 & $0.83 \pm 0.06$ & $0.98 \pm 0.06$ \\
\hline
\end{tabular}


Table 4 Insulin increases the mobility of munc18c in an $80 \mathrm{~K}-\mathrm{H}$ dependant manner

\section{in 3T3-L1 adipocytes}

The indicated EYFP-tagged munc18c constructs were expressed in the presence or absence of $80 \mathrm{~K}-\mathrm{H}$. Cells were serum starved for $24 \mathrm{~h}$ and stimulated with or without $10^{-7}$ M insulin for $20 \mathrm{~min}$. FCS data was collected, diffusion coefficients measured and the effect of stimulation by insulin on the mobility (speed) of the EYFP construct determined. Each value is the ratio of the diffusion coefficients (absence of insulin / presence of insulin). Results are mean \pm s.e.m. for 3 separate experiments each using 6 separate cells. ${ }^{*} \mathrm{p}<0.01$ versus no $80 \mathrm{~K}-\mathrm{H}$. MFL, munc18c full length, M295, munc18c 295; M338, munc18c 338.

\begin{tabular}{lcc}
\hline EYFP & No $80 \mathrm{~K}-\mathrm{H}$ & $80 \mathrm{~K}-\mathrm{H}$ \\
\hline & \multicolumn{1}{c}{ Effect of insulin on mobility } \\
MFL & $1.06 \pm 0.04$ & $2.17 \pm 0.48^{*}$ \\
M295 & $1.12 \pm 0.16$ & $2.51 \pm 0.37^{*}$ \\
M338 & $1.05 \pm 0.36$ & $1.16 \pm 0.10$ \\
\hline
\end{tabular}


Figure. 1

A

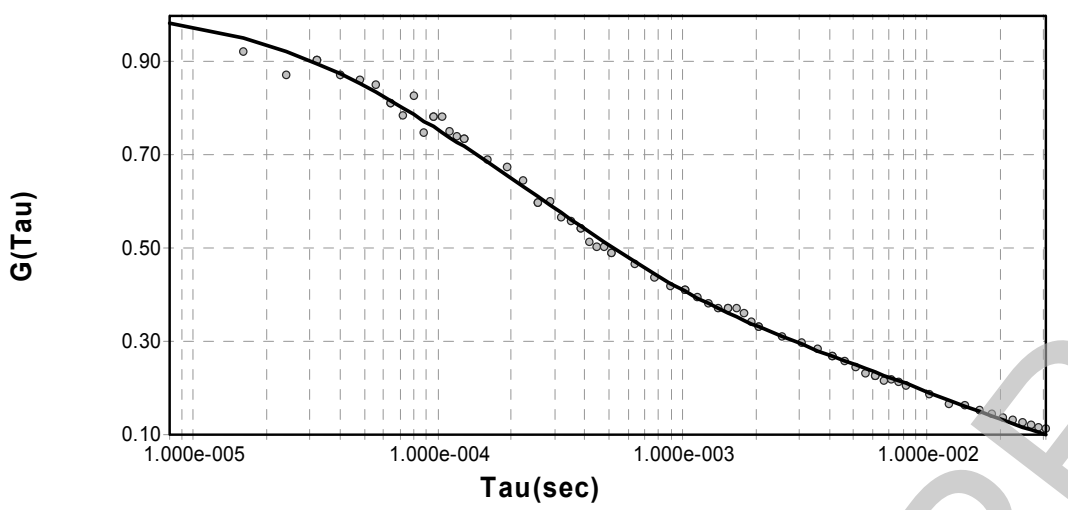

B

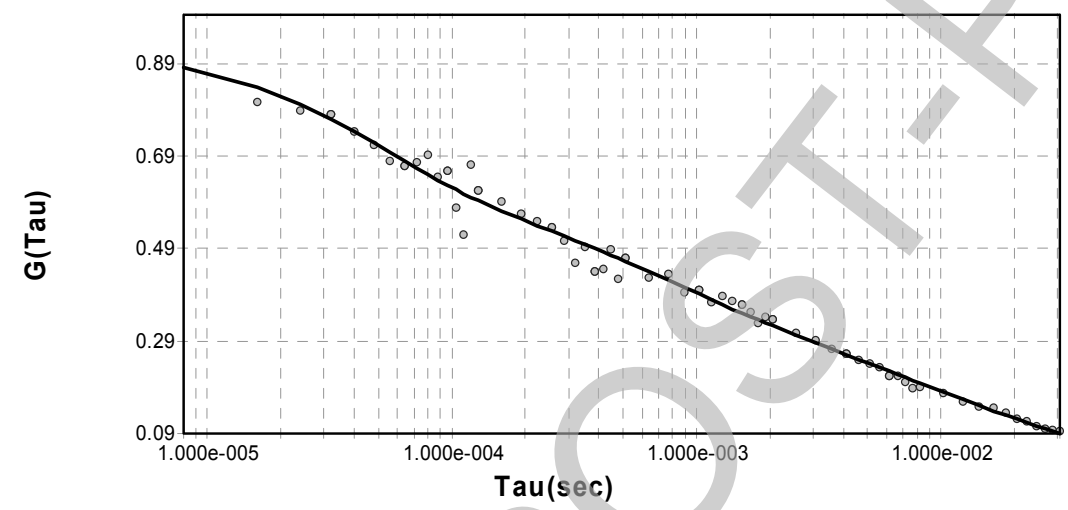

C
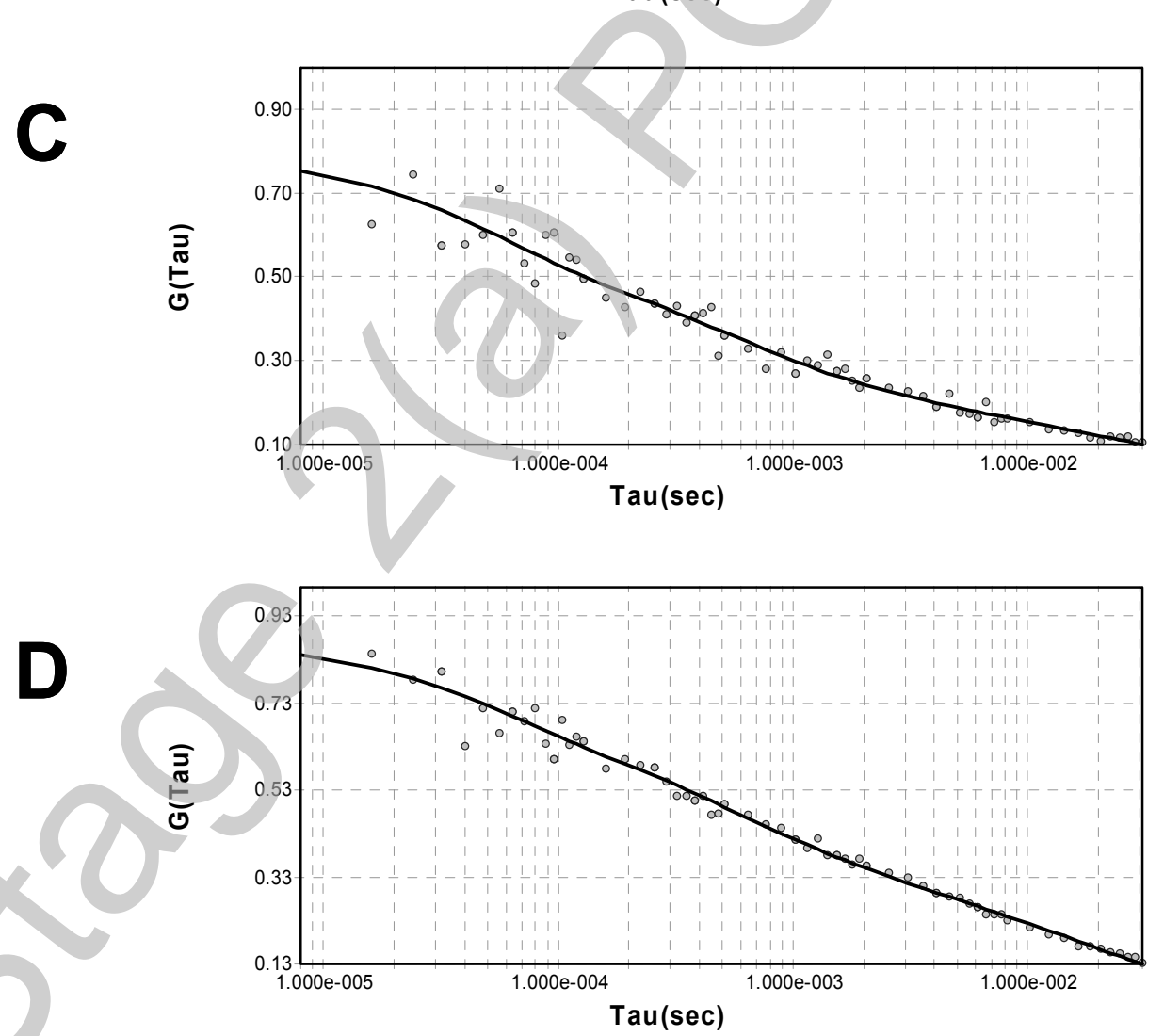
Figure 2

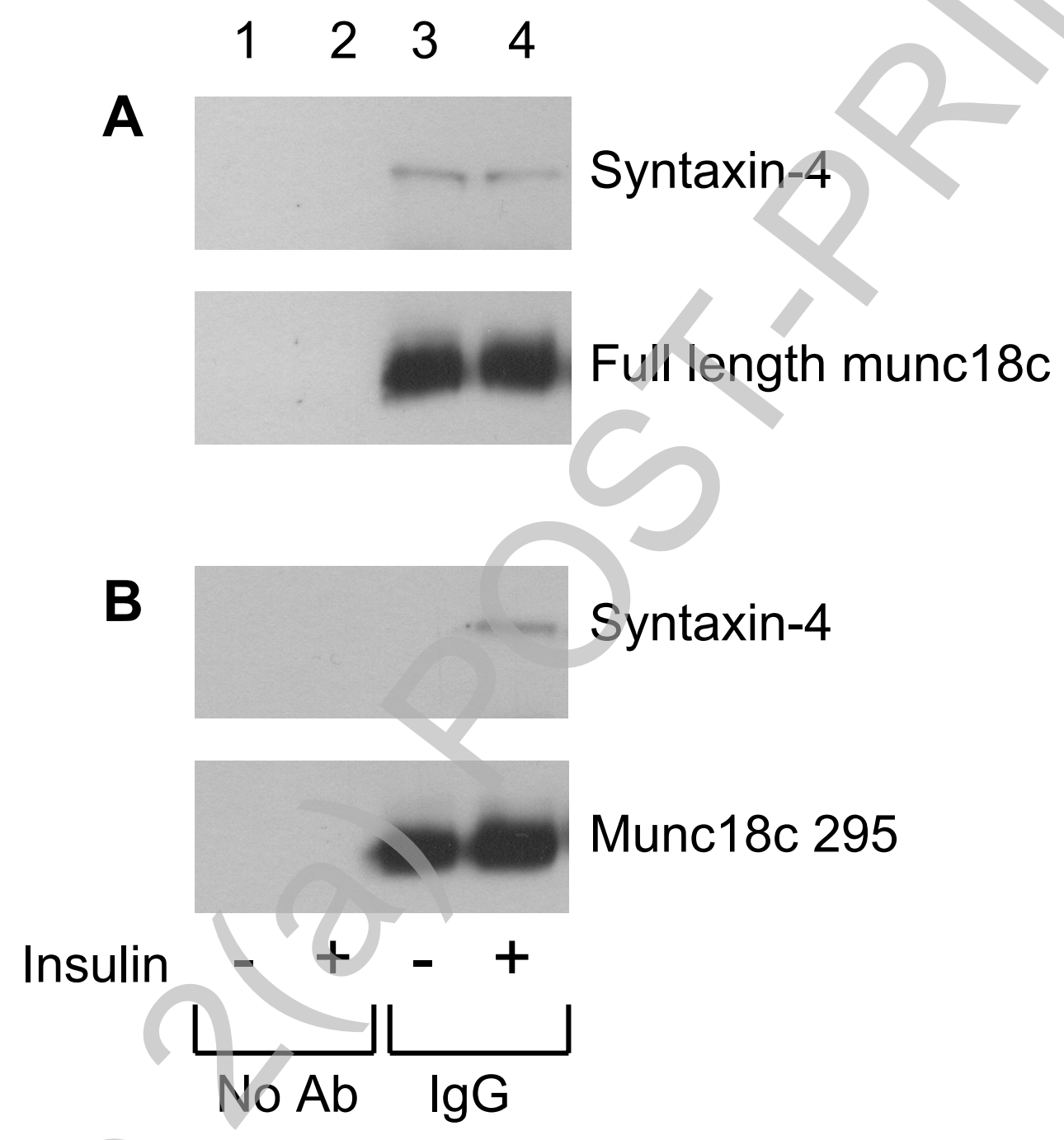


Figure 3

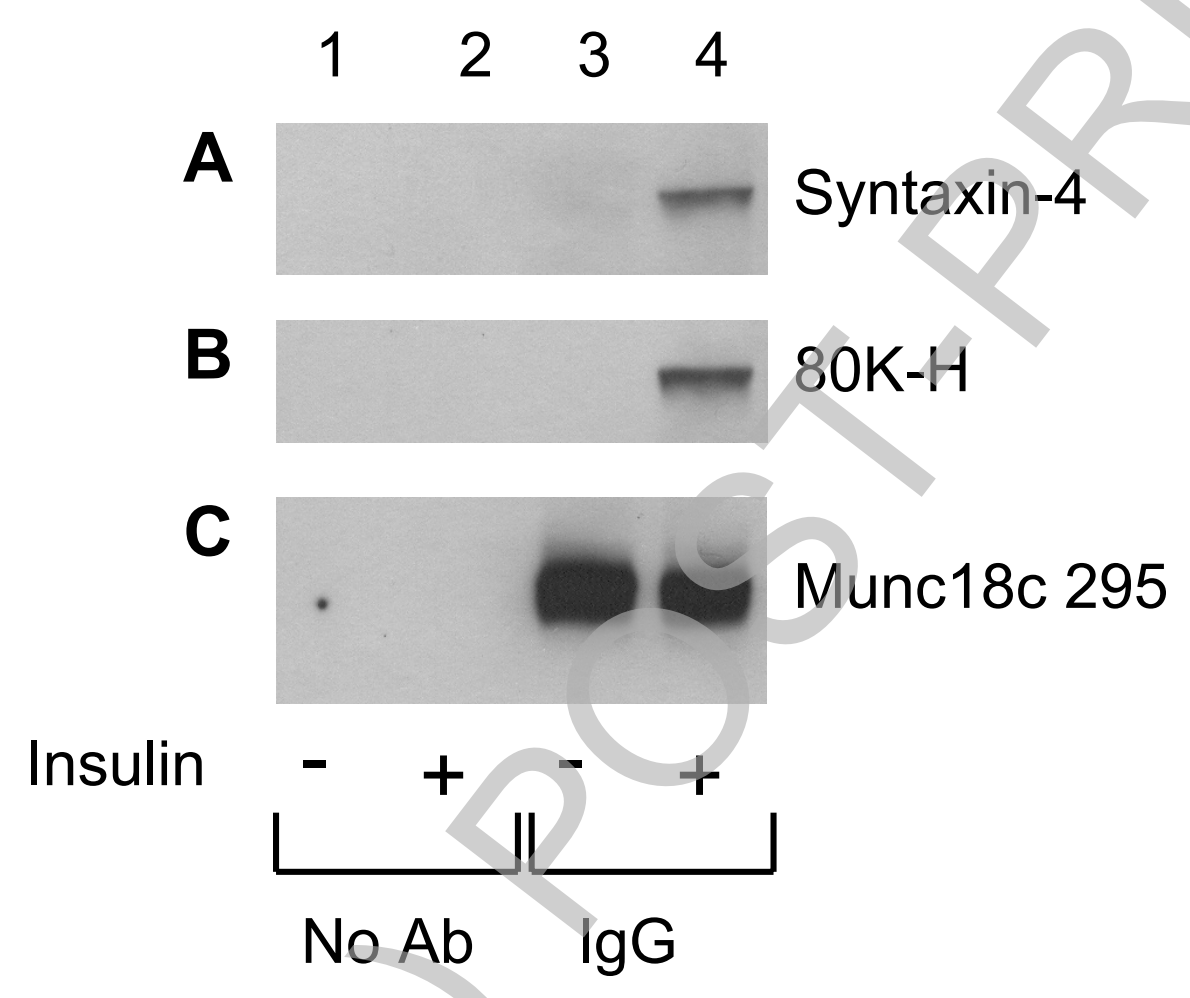


Figure 4

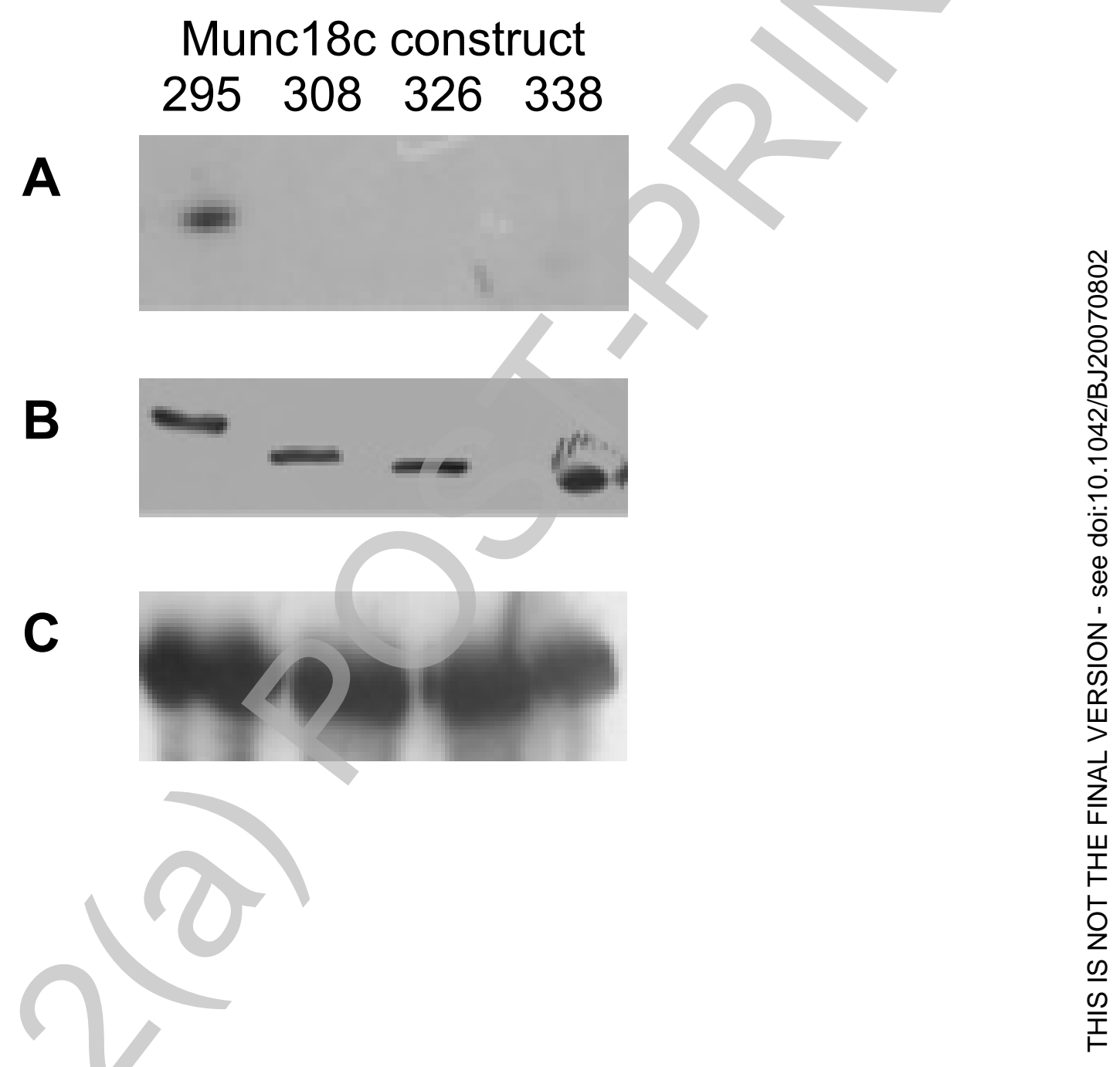


Figure 5

No insulin

GLUT4 vesicle

VAMP-2

\section{Insulin}
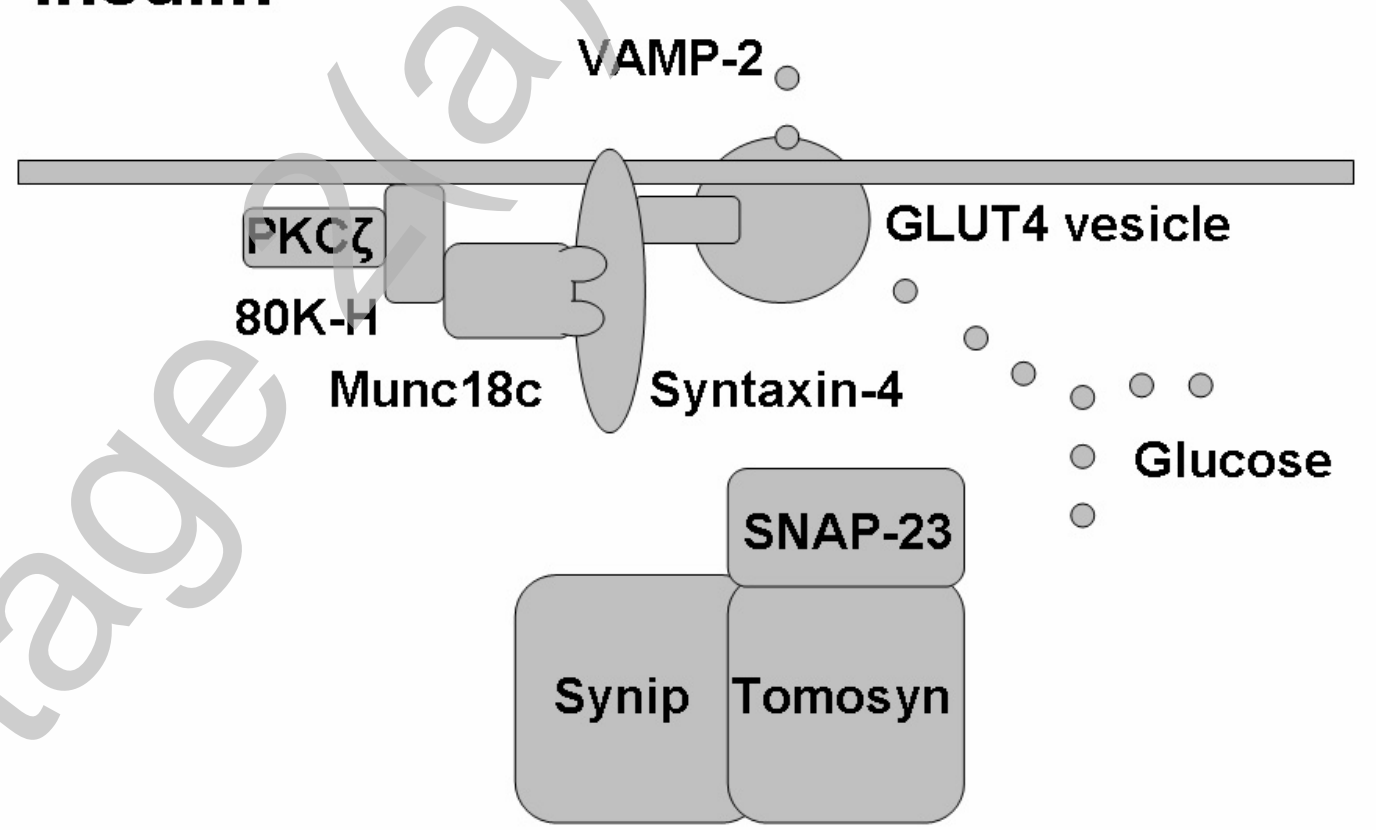